\title{
Conceptual connections between realistic and abstract pictures
}

\author{
RICHARD P. HONECK, TAMMY J. S. CASE, and MICHAEL FIRMENT \\ University of Cincinnati, Cincinnati, Ohio
}

\begin{abstract}
On each of 11 trials, an experimental group was presented a realistic picture (of the literal information in a proverb) and two abstract, nonrepresentational pictures. The subjects chose the abstract picture that provided the best conceptual match for the realistic picture. Control subjects chose from the abstract pictures without having seen the realistic pictures. The experimental group reliably selected the correct picture on most trials whereas the control group performed at chance. Also, when the experimental subjects' rationales for their choices were appropriate, they chose the correct picture $92 \%$ of the time; inappropriate rationales were associated with a 56\% correct response rate. Apparently, the subjects used analogical formats to map surface information in the two kinds of pictures and connected them via a more fundamental abstract medium.
\end{abstract}

People can reliably connect verbal with nonverbal stimuli even when the connections are based on principles that must be figured out. For example, Verbrugge (1977) provided evidence that abstract line drawings can effectively prompt the recall of metaphors. Even children can match pictures on a metaphorical basis, such as a picture of a nearly spent cigar with that of an old man (Connor $\&$ Kogan, 1980). They can apply double function terms (e.g., hard, cold) appropriately to people (Asch \& Nerlove, 1960), and can match such terms with sounds, faces, and colors (Gardner, 1974). They can also analogize parts of the human body to pictures of trees or mountains (Gentner, 1977).

Honeck, Sowry, and Voegtle (1978) showed that 7-, 8 -, and 9-year-old children could reliably match a proverb with a picture that illustrated the proverb's figurative meaning. Honeck, Voegtle, and Sowry (1981) demonstrated that pictures representing the literal level of a proverb could be matched with verbal instances of the figurative meaning of the proverb. These two studies indicate that some verbal-nonverbal connections can be made only through a highly abstract medium.

The present experiment asked whether subjects can match realistic pictures of the literal meaning of proverbs with abstract pictures that constitute interpretations of the proverbs' figurative meanings. A successful demonstration would imply that even more abstract connections are possible than those implicated in previous studies (Honeck et al., 1978; Honeck et al., 1981), for two reasons. First, in both studies one of the stimuli was verbal and, on one level, relatively concrete. Second, our concrete, realistic pictures are more ambiguous than are concrete sentences (proverbs and their instances).

The authors wish to thank Stacy Martz and Melinda McDonald for running subjects and coding the data. Address all correspondence to Richard P. Honeck, Department of Psychology, Dyer Hall (ML 376), University of Cincinnati, Cincinnati, OH 45221.

\section{METHOD}

\section{Subjects}

Twenty-six women and 14 men served as subjects in the experimental group. Twenty subjects served in the control group. All subjects were students in introductory psychology courses at the University of Cincinnati, and they received points worth course credit for their participation.

\section{Materials}

Thirteen realistic pictures and 13 pairs of abstract pictures were constructed. A sample of these materials is presented in Figure 1.

The realistic pictures portrayed the objects and events mentioned in 13 different proverbs at the literal level, 2 practice and 11 test items. In part, these proverbs were selected because they minimized the time element, something not easily represented in a single, static picture. A professional artist composed the pictures with black ink on white bond paper, drawing only the things actually mentioned in the proverb. Most of the pictures had been used by Honeck et al. (1981). In that study, 10 subjects had rated the pictures on a 9point scale in terms of how well the pictures portrayed the objects and events actually mentioned in the proverb. All of the pictures except three received mean ratings of 6.10 or more. Two of these had means of 5.50 and 5.60, but were improved for present purposes. One picture had a mediocre rating of 4.60 . All pictures, including the abstract pictures, were made into transparencies so that they could be shown on an overhead projector.

For each of the 13 proverbs, an abstract picture was drawn that illustrated the figurative meaning of the proverb. The only operational ground rules were that a highly consensual interpretation of each proverb was used as a guide and that "meaningless" lines, angles, forms, and so forth were used, but no common objects. The interpretations were constructed from normative interpretations written by subjects in prior studies (Honeck, Firment, \& Kibler, 1987; Honeck, Kibler, \& Sugar, 1985). The most frequently used elements were circles, squares, triangles, and polygons. All of the pictures conveyed time from left to right in comic-strip-like frames that were separated by vertical double lines. Succeeding frames were used to convey changes in state or processes of various kinds. "Good" things were typically symbolized by symmetrical figures, less good things by misshapen, complex, asymmetrical figures. The experimenters first independently drew the pictures, then chose the best for each proverb and revised it. The final version of each picture was drawn in black ink on white paper, using templates to standardize the drawing process. 
Fan: A fog cannot be dispelled by a fan. To be effective, a solution to a problem must be appropriate to the problem.
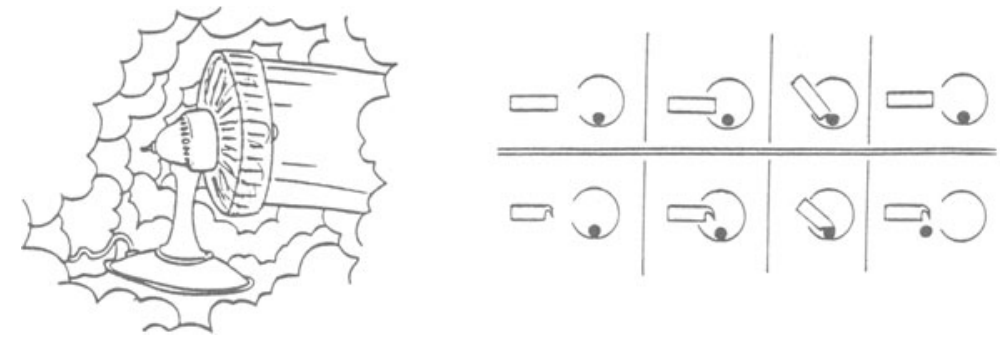

Monkey: The monkey takes the chestnuts out of the fire with the dog's paw. People may use others for their own gain.

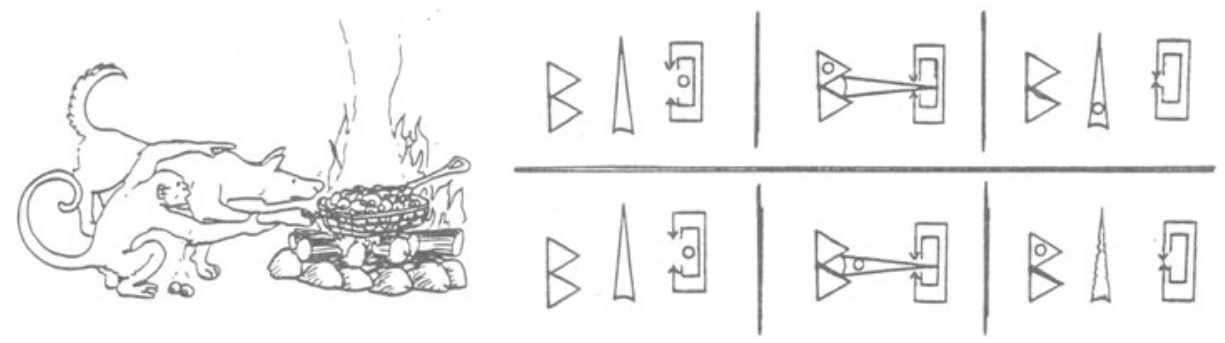

Figure 1. Sample of picture materials. The first sentence is the proverb, the second its interpretation. The picture on the left is the literal picture, the pictures on the right are the abstract pictures. Subjects saw the pictures only.

The target or correct abstract pictures were then used as a basis for drawing companion, but incorrect, pictures. The same criteria and procedures were used to draw these pictures, except that the figurative meaning was not portrayed. Usually, this was accomplished by using the same elements present in the correct picture, but rearranging or otherwise changing their relationship. The pictures in each pair were drawn on the same piece of paper, one above the other and separated by two long horizontal lines, and then copied onto transparencies.

\section{Procedure}

The members of the experimental group were instructed that they would see some pictures on a wall, a common or realistic picture on the left and two abstract pictures on the right. The subjects were told that the realistic picture shared an underlying idea or meaning with one of the abstract pictures. For all problems, the literal and abstract pictures were presented simultaneously for 2 min using two projectors. The subjects circled top or bottom on their answer sheets and then wrote a brief rationale for their choice. For the two practice problems only, the experimenter pointed out the correct picture and then explained why it was correct.

The 11 test problems were presented in three random orders with 25 subjects run on one order, 8 on a second, and 7 on a third. For a particular problem, the two abstract pictures always appeared in the same top/bottom arrangement, but across problems the correct choice appeared on top five times and on bottom six times. All subjects received all of the problems. An experimental session lasted approximately $35 \mathrm{~min}$.

A control group was also run, in order to determine whether there was any a priori preference for one abstract picture over its mate. They were told what the experimental group's task was, but that they, as control subjects, would not see the concrete picture, but were to choose the abstract picture they thought the experimental group had chosen. The control subjects were given $1 \mathrm{~min}$ to select one picture from each pair and to mark A (top picture) or B (bot- tom picture) on their answer sheets. Two random orders of the picture pairs were run, 13 subjects in one order and 7 in the other. Like the experimental subjects, the control subjects were run in small groups.

\section{RESULTS}

\section{Control Data}

Using the $z$-distribution approximation to the binomial, it was determined that preference for a picture was significantly greater than chance $(p<.05)$ if at least $72 \%$, or 14.40 , of the 20 subjects, chose it. Only two (target) pictures were significantly preferred.

\section{Experimental Data}

Overall performance. The maximum possible number of correct responses was 440 . The actual number correct was 298 , which was significantly above chance $(z=$ $5.46, p<.05)$.

Problem-by-problem performance. For each problem, at least $63 \%$, or 26 , of the 40 subjects had to be correct for performance to exceed chance ( $p<.05$, onetailed $z$ test). All but one problem met this criterion, although the incorrect picture was chosen significantly often in one case.

Individual performance. A subject needed to be correct on at least $82 \%$, or 9 , of the 11 problems to show above-chance performance ( $p<.05$, one-tailed $z$ test). Eleven subjects met this criterion. However, an additional 10 subjects had 8 problems correct. No subject had less than 5 problems correct. 
Subjects' rationales. The subjects were asked to explain their choices of pictures. Their written rationales were first coded for appropriateness, that is, whether they were consistent with the interpretation intended by the experimenters. To be considered consistent, a rationale had to relate the correct description of the literal picture to the correct elements in an abstract picture. Subjects' rationales were scored blind to the correctness of the subjects' choices.

We determined that 147 , or $33 \%$, of the rationales were appropriate. More important, the relationship between appropriateness and correct picture choice was strong: the probability of a correct response, given that a rationale was appropriate, was .92 ; the probability of a correct response, given that a rationale was not appropriate, was .56 , or near chance.

The subjects' matching strategies, as suggested by their rationales, consisted of mapping analogies from the literal meaning of the literal pictures to the abstract pictures. Also, five problem sets produced less than $20 \%$ appropriate rationales. This happened because the subjects could not decode the experimenter's intended meaning of either the literal picture or the abstract pictures or both.

\section{DISCUSSION}

Seven of the 11 problem sets were solved on a conceptual basis. This result, along with those for overall and individual performances, warrants the conclusion that subjects can match a picture of the literal things mentioned in a proverb with an abstract picture that was designed to express the figurative meaning of the proverb.

What importance should be attributed to this conclusion? There are two answers, one empirical, the other theoretical. We know of no other demonstration that literal/realistic and abstract pictures can be matched on nonsurface levels. Connor and Kogan (1980), showed that children typically matched two realistic pictures on a metaphorical basis-for example, a baby was matched with a tulip about to open up. In one case, an angry man was matched with dark, haphazard paint strokes. However, the meanings or metaphorical grounds in Connor and Kogan's pictures seem to be resolvable as single concepts-growth, anger, and so onwhereas more complex relational elements connected our pictures. For example, in the monkey problem, the monkey in the literal picture is using the dog to get the "goodies" in the fire. The monkey ends up with the goodies, and the dog ends up hurt. These ideas develop in sequence over the frames in the correct abstract picture-the lower figure uses the triangle to get the circle (in Frame 2), but the triangle ends up deformed (in Frame 3).

Apparently, the subjects had to construct meaning(s) for the literal picture, then scan the abstract pictures and integrate the pictures' statically presented elements and processes. The subjects then had to map the components of the literal picture meaning onto the elements and processes in the abstract pictures. Literal picture meanings apparently drove the search for analogous meanings in the abstract pictures, but the meanings of the two abstract pictures undoubtedly helped shape literal picture meanings as well as the meanings attributed to the abstract pictures themselves.

If our subjects could rationalize their choices better-by implication, if their understanding of the relationship between the pictures was better-they were much better able to choose the correct abstract picture. This corroborates Gentner's (Gentner, 1983; Gentner \& Toupin, 1986) claim that high-order knowledge about a base (literal pictures in our experiment) facilitates mapping to a target domain (our abstract pictures). Clearly, certain attributes of the objects and events in the literal picture had to be canceled in mapping literal meaning to abstract meaning. However, what is the medium in which the constructed shared meaning of concrete and abstract pictures is resolved?
We do not believe the medium is verbal or imaginal. Both of these media must be interpreted to function as representational devices, so the focus then shifts to the interpretation. The medium is not obviously propositional, since it is not clear what it means to say that propositions have meanings (see Honeck \& Kibler, 1985; Honeck et al., 1982). Surely, the medium must be sufficiently abstract, general, and cohesive to allow for the incorporation of inputs from different modalities (pictures, sentences, etc.) and semantic domains. The basic medium functions, therefore, as the core of a category and serves to organize various inputs. We have called the core a conceptual base and have proffered a great deal of evidence for it (see Honeck et al., 1985). More recent evidence comes from Feldhaus (1987), who demonstrated that subjects can use abstract pictures as a guide to consensually rank a set of proverb instances in terms of their conceptual relatedness to the pictures. Moreover, these rankings were significantly correlated with those generated by subjects who used either a proverb or its interpretation as a guide in the Honeck et al. (1985) study.

A final methodological note. Future experiments should examine more carefully the semantic consensualness and clarity of both the concrete and the abstract pictures. For several of our problem sets, one or both kinds of pictures could be interpreted in a variety of ways. Such ambiguity makes the analogy mapping process more difficult. Also, the components of the semantic matches might be laid out in more detail, so that the reasons for unsuccessful matches become more evident. Finally, additional controls may be added, such as random pairing of abstract picture sets with literal pictures.

\section{REFERENCES}

Asch, S., \& Nerlove, H. (1960). The development of double function terms in children. In B. Kaplan \& S. Wapner (Eds.), Perspectives in psychological theory. New York: International Universities Press. ConNor, K., \& KogaN, N. (1980). Topic-vehicle relations in metaphor: The issue of asymmetry. In R. P. Honeck \& R. R. Hoffman (Eds.), Cognition and figurative language (pp. 283-310). Hillsdale, NJ: Erlbaum.

Feldhaus, R. (1987). On the interpretative basis of the gradedness of abstract categories. Unpublished master's thesis, University of Cincinnati, Cincinnati, $\mathrm{OH}$.

GARDNER, H. (1974). Metaphors and modalities: How children project polar adjectives onto diverse domains. Child Development, 45, 84-91.

GENTNER, D. (1977). Children's performance on a spatial analogies task. Child Development, 48, 1034-1039.

GeNTNER, D. (1983). Structure-mapping: A theoretical framework for analogy. Cognitive Science, 7, 155-170.

GENTNER, D., \& TouPIN, L. (1986). Systematicity and surface similarity in the development of analogy. Cognitive Science, 10, 277-300.

Honeck, R. P., Firment, M., \& Kibler, C. (1987). Context and the generalizability of conceptually based categories. Unpublished manuscript, University of Cincinnati, Cincinnati, $\mathrm{OH}$.

HONECK, R. P., \& KIBLER, C. (1985). Representation in cognitive psychological theories of figurative language. In W. Paprotté \& R. Dirven (Eds.), The ubiquity of metaphor: Metaphor in language and thought (pp. 381-423). Philadephia: Benjamins.

HonecK, R. P., Kibler, C., \& SUGAR, J. (1985). The conceptual base view of categorization. Journal of Psycholinguistic Research, 14, 155-174.

Honeck, R. P., Sowry, B., \& Voegtle, K. (1978). Proverbial understanding in a pictorial context. Child Development, 49, 327-331.

Honeck, R. P., Sugar, J., \& Kibler, C. (1982). Stories, categories, and figurative meaning. Poetics, 11, 127-144.

Honeck, R. P., Voegtle, K., \& Sowry, B. (1981). Figurative understanding of pictures and sentences. Journal of Psycholinguistic Research, 10, 135-153.

VERBRUGGE, R. R. (1977). Resemblances in language and perception. In R. E. Shaw \& J. D. Bransford (Eds.), Acting, perceiving, and comprehending: Toward an ecological psychology. Hillsdale, NJ: Erlbaum.

(Manuscript received for publication April 25, 1987.) 